Applied Mathematical Sciences, Vol. 7, 2013, no. 100, 4981 - 4999

HIKARI Ltd, www.m-hikari.com

http://dx.doi.org/10.12988/ams.2013.37428

\title{
Learning and Holding Periods for Portfolio Selection Models: A Sensitivity Analysis
}

\author{
Francesco Cesarone \\ Department of Business Studies \\ University of Rome III, Roma, Italy \\ francesco.cesarone@uniroma3.it \\ Andrea Gheno \\ Department of Business Studies \\ University of Rome III, Roma, Italy \\ andrea.gheno@uniroma3.it
}

Fabio Tardella

Department of Methods and Models for Economics, Territory and Finance Sapienza University of Rome, Roma, Italy

fabio.tardella@uniroma1.it

Copyright (c) 2013 Francesco Cesarone, Andrea Gheno and Fabio Tardella. This is an open access article distributed under the Creative Commons Attribution License, which permits unrestricted use, distribution, and reproduction in any medium, provided the original work is properly cited.

\begin{abstract}
A key issue for applying portfolio selection models in practice is an appropriate choice of learning and holding periods. For this reason some studies in the literature have been devoted to the sensitivity of the selected portfolio performance with respect to either the learning or the holding period. In this paper, an empirical sensitivity analysis of two well-known portfolio selection models is performed for the first time with respect to both the learning and the holding period. In particular, we investigate the outcomes of the Mean-Variance model and of
\end{abstract}


the Mean-Conditional Value-at-Risk model with respect to the length of learning and holding periods for different risk strategies and different rolling time-windows. Weekly data from USA and European stock markets are used, and the influence of the learning data on the portfolio diversification is also examined.

Mathematics Subject Classification: 90C31, 90C90, 91G10

Keywords: Portfolio selection, risk measures, sensitivity analysis, learning period, holding period, diversification

\section{Introduction and motivation}

The selection of an appropriate asset portfolio may be considered as the fund management key activity. Although a significant proportion of portfolio decisions are taken on a qualitative basis, a quantitative approach to selection is often very useful. Harry Markowitz is regarded as the father of modern portfolio theory for his Mean-Variance (MV) portfolio selection model (Markowitz $[18,19,20])$ which is still widely used to support portfolio selection decisions. Some researchers have introduced alternative and often linear risk measures, leading to a simplification from the computational viewpoint. For instance, Konno [15] has proposed the Mean Absolute Deviation (MAD) model, while Speranza [27] has considered the mean absolute value of negative deviations. Roy [26] has laid the seed for the development of portfolio selection models based on downside risk measures where the objective is the maximization of the probability that the portfolio return is above a certain minimal acceptable level. Also Markowitz [19] has proposed the semi-variance as an alternative risk measure, but he has observed that the use of variance is computationally more tractable and reveals the same information.

In the 1970s, several articles (see, e.g., Bawa [6] and Fishburn [12]) have provided a natural generalization of semi-variance by means of the lower partial moment risk measure, arguing that an investor's true risk is the downside risk. In the 1990s J.P. Morgan [22] has proposed what is probably the most famous downside risk measure: the Value-at-Risk (VaR). Following Hong and Liu [14] the $\alpha$-VaR of a random loss is defined as the $\alpha$-quantile of the loss, where $0<\alpha<1$ is typically close to one. Although VaR is a very important risk management tool in the financial industry, it has some drawbacks: for instance, it is not subadditive, i.e., it does not express the benefits of diversification, it does not measure the worst-case losses, and the general VaR optimization problem is not convex. Subadditivity is one of a set of desirable properties that characterize the so-called coherent risk measures (see Artzner et al. [4] for downside risk measures and Rockafellar et al. [25] for dispersion 
measures).

One of the most important coherent risk measures is the Conditional Value-atRisk (CVaR, see Rockafellar and Uryasev [24]), also called Expected Shortfall (see, e.g., Acerbi and Tasche [2]). The $\alpha$-CVaR of a random loss is the average of all $\beta$-VaR of the losses with $\alpha \leq \beta \leq 1$, where $0<\alpha<1$ is typically close to one. Enhanced CVaR measures have been suggested by Mansini et al. [17], Tasche [28] and Acerbi [1]. In spite of the amount of research on risk measures and mean-risk models, the question of which risk measure is most advantageous is still the subject of intense studies. In this paper we focus on variance and $\mathrm{CVaR}$, that certainly play a central role in this debate.

Another related strand of research concerns the analysis of the performance of the efficient portfolios obtained by different models, particularly with respect to the learning and the holding periods used. Lee [16] has observed that a long investment horizon should be used when selecting a portfolio via the MV model. Best and Grauer [7] have used a parametric quadratic programming framework to perform a sensitivity analysis of the MV model. Ulucan [29] has examined the optimal investment horizon for the MV model concluding that the best performances are in general obtained using a holding period of 9 months. Hong and Liu [14] have provided an estimation of sensitivities of CVaR using Monte Carlo Simulation. In an example they have shown that the outcome of the CVaR model is only slightly sensitive to the estimation error in the expected return of assets, while changes in a tail parameter for heavy-tailed distribution can lead to large variations of the results. DeMiguel et al. [10] have compared the performance of the MV model (and its extensions) with that of equally-weighted portfolio finding that the performance of the latter is almost always better than that of the other portfolios.

Our work belongs to this latter strand of research. In fact we investigate for the first time the performance of the MV model and of the Mean-Conditional Value-at-Risk (MCVaR) model by varying both the learning and the holding period for different risk strategies and different rolling time-windows. Weekly data from four stock markets (Europe, UK, Italy and USA) are used and the influence of the learning data on the portfolio diversification is also examined. Furthermore, the performances of the selected portfolios are compared to those of the equally-weighted portfolio and of the market index, chosen as benchmarks.

The paper is organized as follows: Section 2 introduces the mean-risk models. In Section 3 we explain how we perform the study and describe the data sets considered. Section 4 reports the main results of the analysis made both with respect to the performance and to the diversification induced by the models. The conclusions of our study are drawn in Section 5. 


\section{Mean-risk models}

\subsection{The MV model}

The classical MV portfolio optimization model introduced by Markowitz aims at determining the fractions $x_{i}$ of a given capital to be invested in each asset $i$ belonging to a predetermined set. The objective is to minimize the risk of the whole portfolio, measured by the variance of its return, while restricting the expected return to a specified level. The MV model is thus based on the estimates of the means and covariances of the assets returns and therefore several methods have been proposed to infer them (see, e.g., Meucci [21] and references therein). Such estimates clearly depend on the length $\Delta L$ of the period considered in which we observe the assets returns that are viewed as realizations of the corresponding random variables. This period is usually called in-sample or learning period. In particular, for a learning period of length $\Delta L$ we denote by $\mu_{i}(\Delta L)$ the estimate of the expected return of asset $i$ and by $\sigma_{i j}(\Delta L)$ the estimate of the covariance of returns of asset $i$ and asset $j$. Thus for a required level $\rho$ of the portfolio expected return the classical MV model can be represented as follows:

$$
\begin{array}{lll}
\min _{x} & \sum_{i=1}^{n} \sum_{j=1}^{n} \sigma_{i j}(\Delta L) x_{i} x_{j} & \\
\text { st } & & \\
& \sum_{i=1}^{n} \mu_{i}(\Delta L) x_{i}=\rho & \\
& \sum_{i=1}^{n} x_{i}=1 & i=1, \ldots, n
\end{array}
$$

where $n$ is the number of assets available in the market. This is a convex quadratic programming problem which can be solved by a number of efficient algorithms with a moderate computational effort even for large instances. Let $\rho_{\text {min }}(\Delta L)$ denote the value of $\sum_{i=1}^{n} \mu_{i}(\Delta L) x_{i}$ at the optimal solution of the problem obtained by deleting the first constraint in $(1)$, and let $\rho_{\max }(\Delta L)=$ $\max \left\{\mu_{1}(\Delta L), \ldots, \mu_{n}(\Delta L)\right\}$. We denote by $\phi(\Delta L, \rho)$ the optimal value of $(1)$ as a function of $\Delta L$ and $\rho$, with $\rho(\Delta L) \in\left[\rho_{\min }(\Delta L), \rho_{\max }(\Delta L)\right]$. Note that the graph of $\phi(\Delta L, \rho)$ on the interval $\left[\rho_{\min }(\Delta L), \rho_{\max }(\Delta L)\right]$ coincides with the set of all non-dominated (or efficient) portfolios (efficient frontier), and is usually approximated by solving (1) for several (equally spaced) values of $\rho$ in $\left[\rho_{\min }, \rho_{\max }\right]$ (see an example in Fig. 1$)$. In order to enhance comparability of our results with those available in the literature, the market parameters $\mu_{i}(\Delta L)$ and $\sigma_{i j}(\Delta L)$ are respectively estimated by sample means and covariances. 


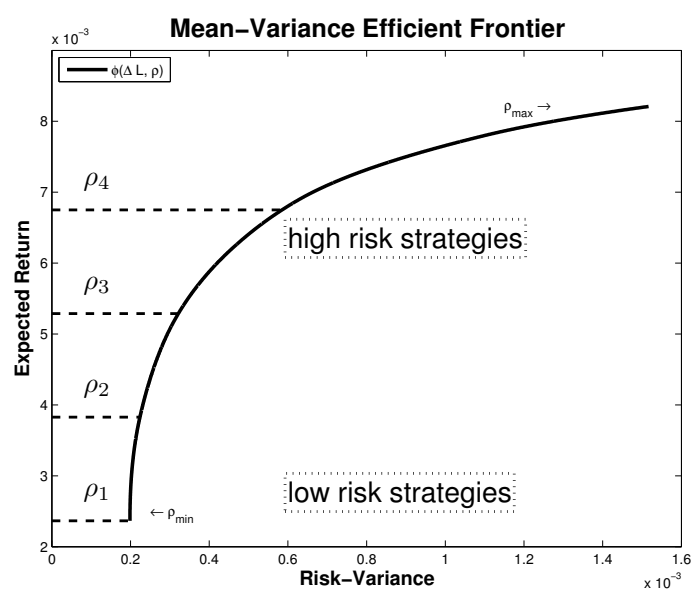

Figure 1: Example of selected target return

\subsection{The MCVaR model}

According to Acerbi and Tasche [2] the CVaR, at the specified significance level $\epsilon$, is the average of losses in the worst $100 \epsilon \%$ of the cases, that is, the mean shortfall at a specified confidence level. In our analysis we set $\epsilon$ equal to 0.05 and losses are defined as negative outcomes. Equality holds if the $\epsilon$-quantile of the portfolio return $R$ is unique, which is always true when $R$ is a continuous random variable. CVaR is known to have better properties than VaR. In fact, Pflug [23] proved that CVaR is a coherent risk measure which also satisfies convexity and monotonicity with respect to first- and second-order stochastic dominance. Formally, the MCVaR model can be written as follows:

$$
\begin{array}{ll}
\min _{x} & C \operatorname{VaR}(x, \epsilon) \\
\text { st } & \\
& \sum_{i=1}^{n} \mu_{i} x_{i}=\rho \\
& \sum_{i=1}^{n} x_{i}=1 \\
& x_{i} \geq 0 \quad i=1, \ldots, n
\end{array}
$$


As shown by Rockafeller and Urysaev [24], in the discrete case and for a given learning period $\Delta L$, problem (2) becomes:

$$
\begin{array}{ll}
\min _{(x, \zeta, d)} \zeta+\frac{1}{\epsilon} \frac{1}{\Delta L} \sum_{t=1}^{\Delta L} d_{t} & \\
d_{t} \geq \sum_{i=1}^{N}-r_{i t} x_{i}-\zeta & \\
d_{t} \geq 0 & \\
\sum_{i=1}^{n} \mu_{i}(\Delta L) x_{i}=\rho & \\
\sum_{i=1}^{n} x_{i}=1 & \\
x_{i} \geq 0 & \\
\zeta \in \mathbb{R} &
\end{array}
$$

where we assume that all scenarios $r_{i t}$, with $i=1, \ldots, n$ and $t=1, \ldots, \Delta L$, are equally likely, i.e., $p_{t}=1 / \Delta L$. The $d_{t}$ are auxiliary variables used to linearize the original objective function and are defined as the deviation of the portfolio losses (negative outcomes), denoted by $l_{t}=\sum_{i=1}^{n} x_{i}\left(-r_{i t}\right)$, from $\zeta$ when $l_{t}>\zeta$ and zero otherwise. Index $j$ represents different scenarios.

Rockafeller and Urysaev [24] also proved that, when returns are normally distributed, the solutions of the portfolio selection problem described by the MCVaR or the MV models are the same. However, in the case of non-normal distribution of returns, the solutions may be significantly different, as also shown in our analysis.

\section{Methodology and data}

\subsection{Definition of the indices and benchmark portfolios}

We investigate the performance of MV and MCVaR models focusing on learning and holding periods. The holding (or out-of-sample) period represents the investment horizon of the selected portfolio. We measure the performance of a portfolio by using a standard Portfolio Index (PI), which provides the ratio between the values of the portfolio at the end and at the beginning of the holding period. Let $p_{i}^{0}$ and $p_{i}^{t}$ denote the prices of asset $i$ at the beginning (time 0 ) and at the end (time $t$ ) of a holding period of length $\Delta H=t$; let $V^{0}$ denote the value of a portfolio at time 0 and let $x_{i}$ be the fraction of such value invested in asset $i$. Then the value $V^{t}$ of the same portfolio at time $t$ is given by

$$
V^{t}=\sum_{i=1}^{n} \frac{V^{0} x_{i}}{p_{i}^{0}} p_{i}^{t}
$$


For a portfolio $x=\left(x_{1}, \cdots, x_{n}\right)$ we consider its PI (see, e.g., Bacon [5])

$$
P I(x, t)=\frac{V^{t}}{V^{0}}=\sum_{i=1}^{n} x_{i} \frac{p_{i}^{t}}{p_{i}^{0}}
$$

and its return $R(x, t)=P I(x, t)-1$ with respect to the holding period considered. We compare the returns of the PIs obtained by the MV and MCVaR models with those of the Market Index (MI) and of the Uniform Portfolio (UP), also called equally-weighted or naïve portfolio, where $x_{i}=\frac{1}{n}$ for all $i$.

\subsection{Data sets and summary statistics}

In our analysis we use historical prices of securities of four stock markets: EuroStoxx50 (Europe), FTSE 100 (UK), MIBTEL (Italy), S\&P 500 (USA). For each stock we have 263 weekly price data for the period from March 2003 to March 2008. The data sets consist of 47 stocks for EuroStoxx50, 76 for FTSE 100, 221 for MIBTEL and 476 for S\&P 500 (for more details see Cesarone et al. [8]).

The return rates are computed as logarithmic variations of the quotation prices $\left(\ln \left(P_{t} / P_{t-1}\right)\right)$. These data sets are available from the authors upon request and their summary statistics are provided in Fig. 2. For each market we report the empirical distribution of the expected weekly returns, of the stock return volatilities and of the linear correlations for all pairs of stocks. The dashed vertical lines represent the 0.5 and 0.95 percentile of each empirical distribution. The EuroStoxx 50 has $90 \%$ of assets with expected weekly returns ranging from -0.0004 to 0.0054 . These securities show the highest correlations $(90 \%$ of them is between $17 \%$ and $61 \%$ ), while their standard deviations are the lowest among the markets analyzed, ranging from 0.0243 to 0.0435 . 90\% of FTSE 100 assets exhibit expected returns between -0.0007 and 0.0069 , thus higher than the EuroStoxx50 ones. Furthermore, the volatility range is [0.0227; 0.0528], while $90 \%$ of correlations between pairs of securities are between $5 \%$ and $47 \%$, thus shifted to the left with respect to EuroStoxx50. The data sets with more securities show fat tailed empirical distributions of expected returns and standard deviations. For MIBTEL, 90\% of assets have expected returns between -0.0039 and 0.0057 and volatilities between 0.0250 and 0.0737 , while for S\&P 500 the ranges are $[-0.0020 ; 0.0071]$ and $[0.0230 ; 0.0606]$, respectively. Note that MIBTEL presents the widest interquantile range of standard deviations and the tightest range for the distribution of correlations $(5 \%-33 \%)$, which is also lower than that of the other markets. For S\&P 500, 90\% of pairs of stocks have correlation between $8 \%$ and $45 \%$. 

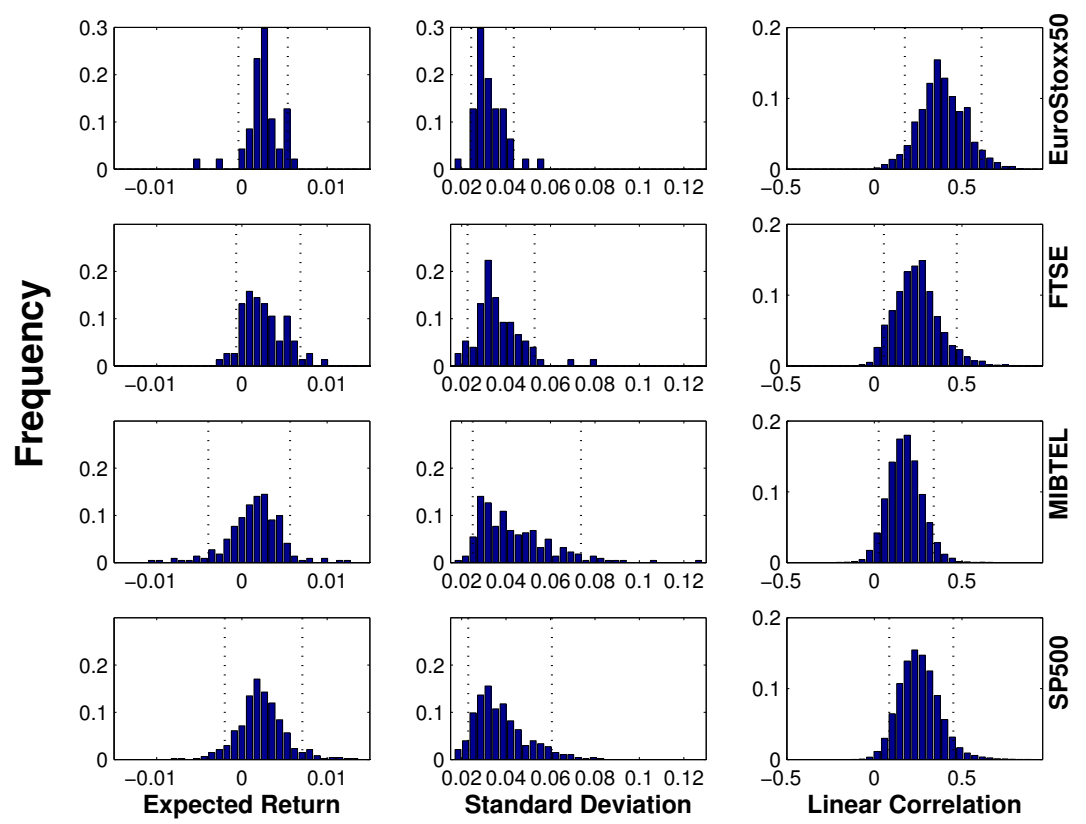

Figure 2: Summary statistics of the assets for the period from March 2003 to March 2008

\subsection{Structure of the analysis}

In our analysis we vary the in-sample period $\Delta L$ between 13 weeks ( $\simeq 3$ months) and 156 weeks ( $\simeq 3$ years), and the out-of-sample period $\Delta H$ between 13 weeks and 91 weeks. Furthermore we perform in-sample and out-of-sample evaluations on several rolling time windows. The rolling time window strategy is sketched in Fig. 3. To characterize different investors attitudes, four different target return levels are fixed (see Fig. 1): $\rho_{1}=\rho_{\min }, \rho_{2}=\frac{1}{4}\left(\rho_{\max }-\rho_{\min }\right)$, $\rho_{3}=\frac{1}{2}\left(\rho_{\max }-\rho_{\min }\right), \rho_{4}=\frac{3}{4}\left(\rho_{\max }-\rho_{\min }\right)$.

The analysis involves solving a considerable number of portfolio optimization problems, a total of 8832 problems that arise by combining four stock markets, four different target return levels, two different risk measures and 276 different sampling periods. The codes are written in MATLAB 7.4 and executed on a workstation with Intel Core2 Duo CPU (T7500, 2.2 GHz, 4Gb RAM) under Windows Vista. CPLEX 11.2 is also called from MATLAB with the TOMLAB/CPLEX toolbox (see Holmstrom et al. [13]). We estimate with respect to the chosen $\Delta L$ period the input parameters of the MV and MCVaR models. Thus, at the beginning of $\Delta H$ (i.e., at the end of $\Delta L$ ) we have the optimal portfolios $x_{M V}^{*}$ and $x_{M C V a R}^{*}$ that we use to compute the Portfolio Indices $P I\left(x_{M V}^{*}\right)$ and $P I\left(x_{M C V a R}^{*}\right)$ and their returns. We use a rolling period of 5 weeks. The number of time windows that it is possible to consider de- 


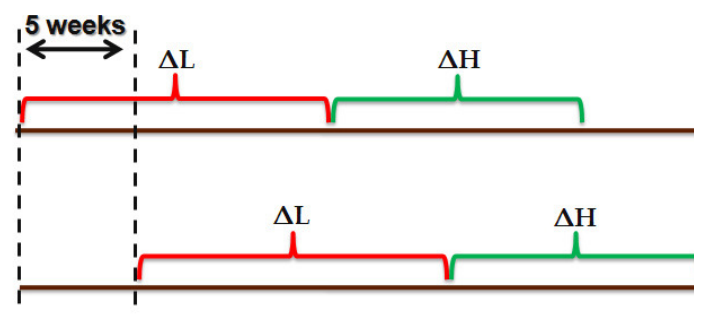

Figure 3: Structure of the rolling time periods used in the analysis

creases with respect to $\Delta L$ and $\Delta H$ and is reported in Table 1 . For example, if $\Delta L=156$ weeks and $\Delta H=91$ weeks we have the minimum number of time windows which is equal to three, while if $\Delta L=\Delta H=13$ weeks, we obtain a maximum of 47 time windows. Thus, for each choice of $\Delta L$ and $\Delta H$ we solve a number of portfolio selection problems and compute the corresponding optimal portfolio returns. We then average such returns to estimate the performance of a model for the selected values of $\Delta L$ and $\Delta H$. In order to make a fair comparison of these average returns related to holding periods $\Delta H$ of different length we also compute in each case the financially equivalent return with respect to the shortest (13 weeks) $\Delta H$ period. Formally we have

$$
E R_{\Delta H}=\left(1+R_{\Delta H}\right)^{13 / \Delta H}-1
$$

where $R_{\Delta H}$ is the average return found with a holding period $\Delta H$ and $E R_{\Delta H}$ is its equivalent return with respect to a holding period of 13 weeks.

\section{Main results}

In this section we discuss the sensitivity analysis of the portfolio models in order to evaluate the impact of the length of the learning $(\Delta L)$ and hold- 


\begin{tabular}{|l||c|c|c|c|c|c|c||}
\hline$\Delta L \backslash \Delta H$ & $\mathbf{1 3 w}$ & $\mathbf{2 6 w}$ & $\mathbf{3 9 w}$ & $\mathbf{5 2 w}$ & $\mathbf{6 5 w}$ & $\mathbf{7 8 w}$ & $\mathbf{9 1 w}$ \\
\hline \hline $\mathbf{1 3 w}$ & 47 & 44 & 42 & 39 & 36 & 34 & 31 \\
$\mathbf{2 6 w}$ & 44 & 42 & 39 & 36 & 34 & 31 & 29 \\
$\mathbf{3 9 w}$ & 42 & 39 & 36 & 34 & 31 & 29 & 26 \\
$\mathbf{5 2 w}$ & 39 & 36 & 34 & 31 & 29 & 26 & 23 \\
$\mathbf{7 8 w}$ & 34 & 31 & 29 & 26 & 23 & 21 & 18 \\
$\mathbf{1 0 4 w}$ & 29 & 26 & 23 & 21 & 18 & 16 & 13 \\
$\mathbf{1 3 0 w}$ & 23 & 21 & 18 & 16 & 13 & 10 & 8 \\
$\mathbf{1 5 6 w}$ & 18 & 16 & 13 & 10 & 8 & 5 & 3 \\
\hline \hline
\end{tabular}

Table 1: Number of windows considered

ing $(\Delta H)$ periods. We compare, on out-of-sample data, the performances of portfolios obtained by the MV and MCVaR models with those of the standard market benchmarks. Furthermore we analyze the diversification of the selected portfolios with respect to the length of the learning period $\Delta L$ and to the level of required return. A synthesis of the main questions that we address is:

1. What is the behavior of the MV and MCVaR portfolio models when varying both the in-sample period $\Delta L$, on which we compute the optimal portfolios, and the holding period $\Delta H$ of the same portfolios? In other words, what are the best patterns of $\Delta L$ and $\Delta H$ with respect to performance?

2. Can we identify any general tendency arising from the comparison between the indices obtained with the models and the market benchmarks?

3. Are there particular patterns of diversification depending on the insample period $\Delta L$ and on the target return level of the portfolio?

\subsection{Performance analysis of the MV and MCVaR mod- els}

We evaluate and compare the performance of the optimal portfolios obtained by the MV and MCVaR models by ex-post analysis. Our procedure consists of the following steps:

1. fix the value of the required return $\rho$, the length of the learning period $\Delta L$ and of the holding period $\Delta H$;

2. for each time-window of length $\Delta L$, compute the efficient frontier by solving the classical Markowitz problem for different return levels and find the target returns $\rho_{1}, \rho_{2}, \rho_{3}, \rho_{4}$ described in Section 2; 
3. for each $t=\Delta L+1, \Delta L+1+5, \ldots, T-\Delta H$ find the optimal portfolios for the return levels $\rho_{1}, \rho_{2}, \rho_{3}, \rho_{4}$ with respect to the observed returns on the time interval $[t-\Delta L, t-1]$. Evaluate the PIs and the returns of the portfolios obtained with these optimal solutions on the time interval $[t, t+\Delta H]$

4. compute the mean of such returns and their financially equivalent returns with respect to the 13 weeks period.

An example of these analyses for equivalent returns $E R_{\Delta H}$ is depicted in Fig.4. In the upper part we report the equivalent returns of the optimal portfolios (MV on the left and MCVaR on the right) on a $\Delta L \times \Delta H$ grid. The lighter the color the lower the return values. At the bottom of Fig. 4, we present the cross-sections of these grids. Specifically each cross-section provides different lines for each value of $\Delta H$, showing the in-sample period $\Delta L$ on the $x$-axis and the equivalent return $E R_{\Delta H}$ on the $y$-axis.

It is apparent that the performance of the portfolio does not depend only on the risk measure used. For instance, for both risk measures, if we focus our attention on $\Delta L=52$, we observe a percentage of performance variation that is up to $50 \%$ for different values of $\Delta H$. Furthermore, the variation of the optimal portfolios performances with respect to $\Delta H$ is generally greater for smaller values of $\Delta L$ and smaller for greater values of $\Delta L$. In other words, the benefit of a longer investment horizon decreases when the learning period increases. This phenomenon is more evident when $\Delta L$ is not greater than 2 years for low risk strategies (see Figs. 4 and 5).

On the other hand, when focusing on each choice of $\Delta H$, we note that the sensitivity with respect to $\Delta L$ is considerable. More precisely, as shown in Figs. 4 and 5, we observe that for fixed $\Delta H$ and for all data sets analyzed the equivalent return tends to decrease noticeably when $\Delta L$ increases above 2 years, particularly for small or medium target return levels.

In Fig. 5 we present the performance of portfolios for higher target return levels $\left(\rho_{2}, \rho_{3}, \rho_{4}\right)$. It seems that for these return levels the performance of the selected portfolios is less sensitive to changes in the length of the in-sample period. This feature is evident both referring to the MV model and to the MCVaR model.

Based on empirical findings, some significant results can be summarized as follows:

1. the MV and MCVaR portfolios have generally better performance in longer-term investment horizons, but it seems that the benefit of increasing $\Delta H$ decreases, when $\Delta H$ is greater than 1 year;

2. the best performance values are generally obtained when $\Delta L$ is between 1 and 2.5 years. This empirical evidence seems to contrast with the 

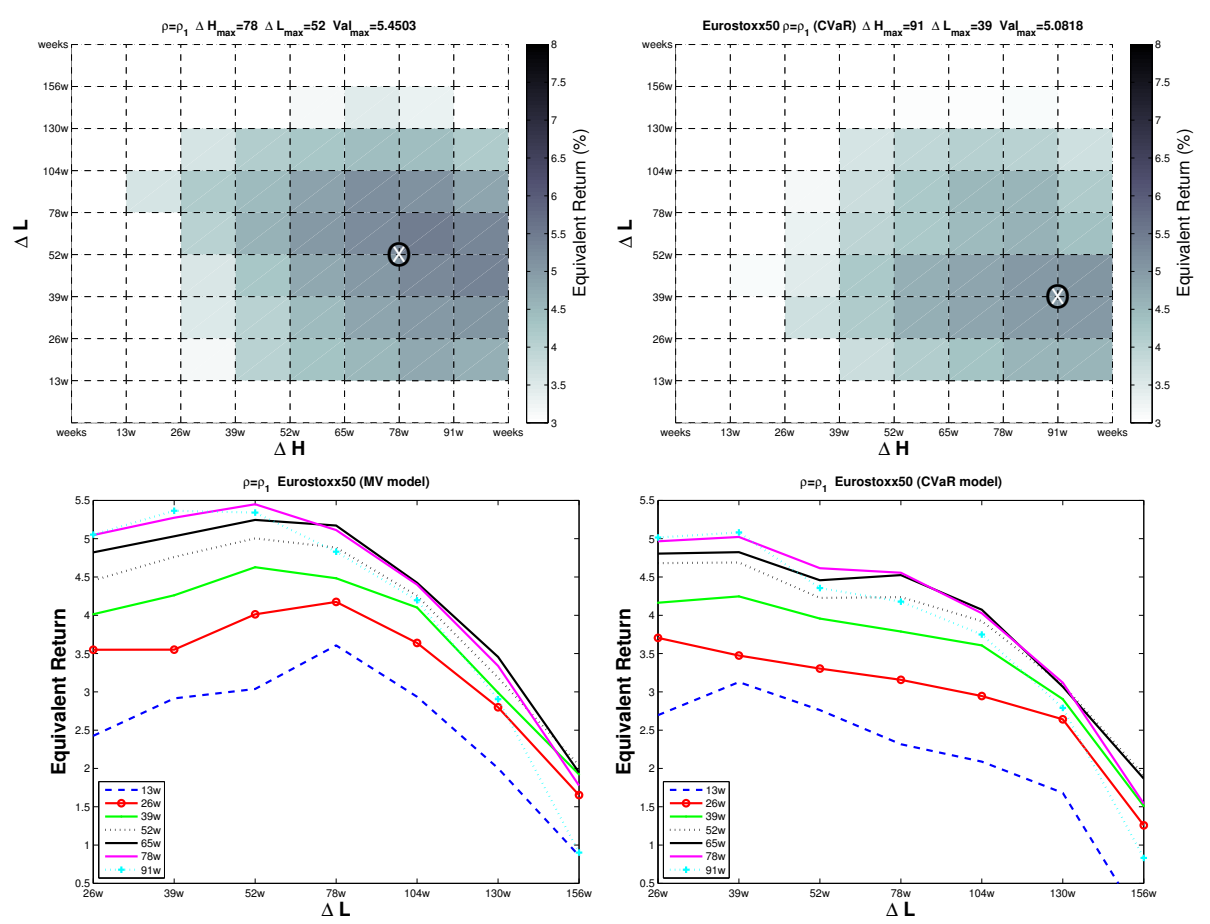

Figure 4: MV and MCVaR PI equivalent mean return by varying $\Delta L$ and $\Delta H$ for $\rho=\rho_{1}$ on a grid (top) and on its cross-section (bottom)

conventional view among practitioners to rely on the past 5 years of data given the belief that such a time interval is generally consistent with a typical economic cycle.

\subsection{Comparison with standard benchmarks}

In this section we compare the performances of the optimal PIs previously analyzed with the standard benchmarks described in Section 3.1. Considering the procedure described in Section 3.3, an example of a comparison of the PI mean return with that of the UP for the FTSE100 data set is reported in Fig. 6.

Based on empirical findings, we observe that for low risk strategies $\left(\rho=\rho_{1}\right)$ the UP seems to outperform the optimal portfolios found by both models for all choices of the learning period $\Delta L$ and of the holding period $\Delta H$. This is consistent with empirical and theoretical findings of DeMiguel et al. [10] who have shown that, in the MV setting, the most pessimistic investors tend to hold more diversified portfolios. On the other hand, we find that for higher risk strategies $\left(\rho \geq \rho_{2}\right)$ the MV and MCVaR models outperform the UP for almost all combinations of $\Delta L$ and $\Delta H$ on all the data sets analyzed. We also find that the MI performance is generally worse than that of the optimal portfolios 

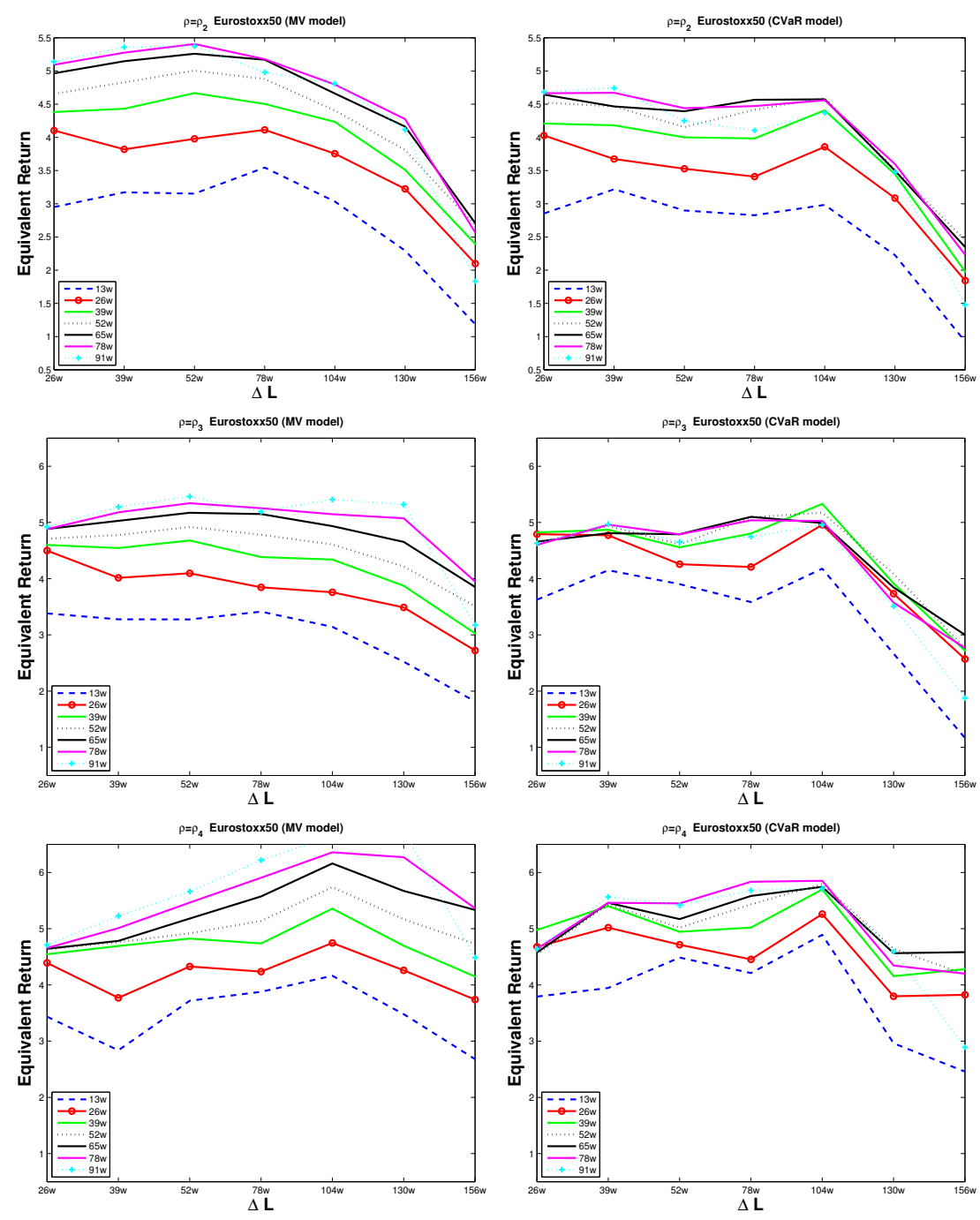

Figure 5: MV (left) and MCVaR (right) PI equivalent returns for $\rho=\rho_{2}, \rho_{3}, \rho_{4}$. 

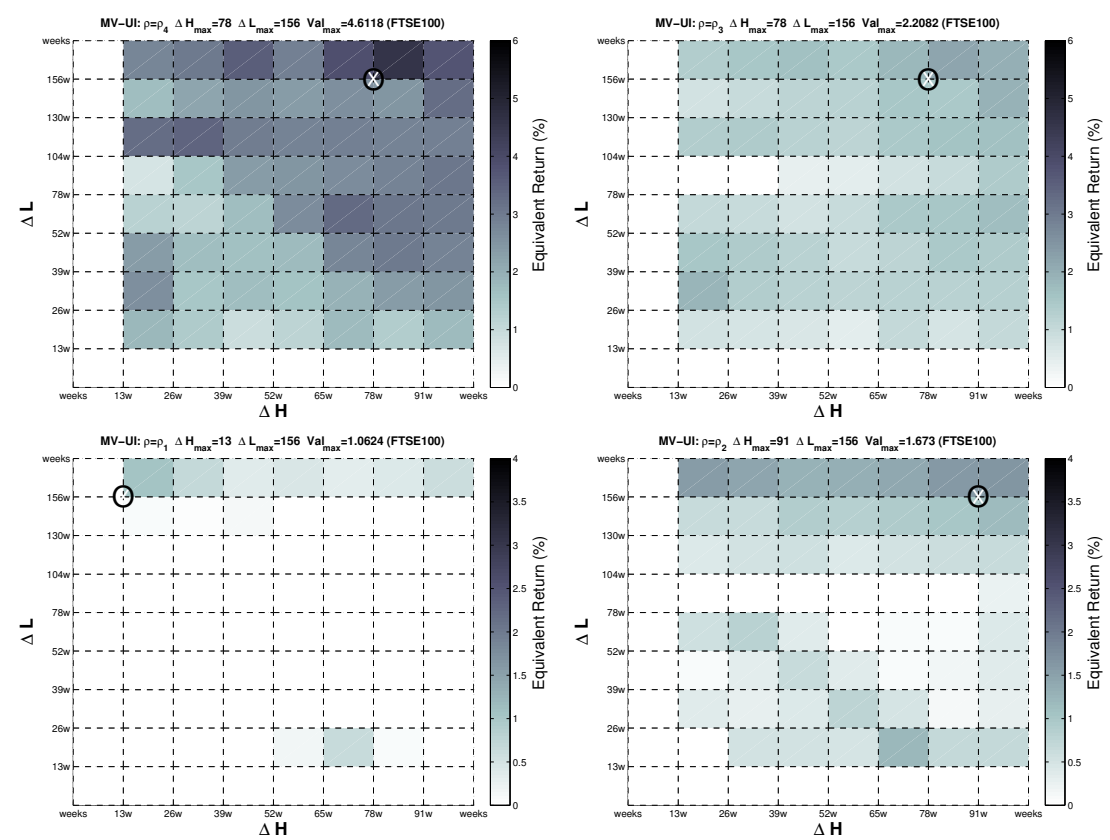

Figure 6: Difference between the MV PI and UI equivalent mean returns for $\rho=\rho_{i}$ with $i=1, \ldots, 4$ on the FTSE100 data set $(\rho$ increases counterclockwise)

provided by the models. An example on the FTSE100 data set is shown in Fig. 7, where we present the differences between the MV equivalent return for the target values $\rho_{1}, \rho_{2}, \rho_{3}, \rho_{4}$ and the MI equivalent return. The white color corresponds to a negative difference, while darker colors correspond to positive differences. Similar results are found with the MCVaR model and are available upon request.

\subsection{Diversification analysis}

As mentioned above (see, in particular, Fig. 6), there is some evidence that for low values of $\rho$ the best portfolio is the UP that equally distributes the overall budget on each asset. Hence it is interesting to study more carefully the behavior of the models with respect to diversification. We investigate the diversification by computing the Herfindal Index (or diversification ratio) $H I(x)=\left(\sum_{i=1}^{n} x_{i}^{2}\right)^{-1}$, which is considered a common measure of diversification (see, e.g., Adam et al. [3]). Higher Herfindal Index values correspond to more diversified portfolios. The structure of the analysis on diversification is similar to the steps described above for evaluating the performance of the models (see Section 4.1). We determine the Herfindal index on rolling time windows 

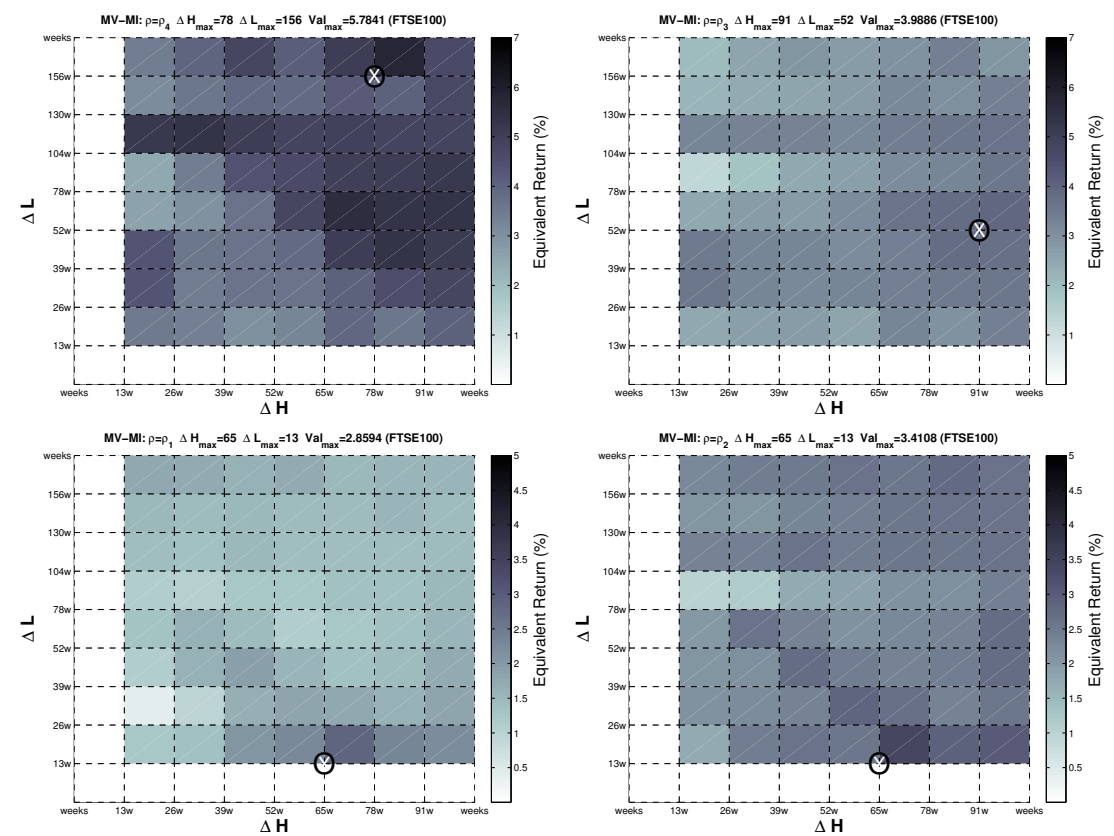

Figure 7: Difference between the MV PI and MI equivalent mean returns for $\rho=\rho_{i}$ with $i=1, \ldots, 4$ on the FTSE100 data set ( $\rho$ increases counterclockwise)

of the same length and we compute the average of these values. We show the evolution of the diversification ratio with respect to $\Delta L$ for the $\mathrm{MV}$ and MCVaR models in Figs 8 and 9, respectively.

From the results we derive that, as expected, the diversification almost always decreases when the target return level increases. Furthermore the MV model generally leads to a more diversified portfolio than the MCVaR model (confirming the diversification effect at the basis of the MV theory). Figs 8 and 9 show that anomalies can occur when the values of $\Delta L$ are low, likely due to estimation errors of the input parameters of the model and to strong short-term non-stationarity. We also observe that for MV efficient portfolios the diversification ratio tends to increase with $\Delta L$. However, when the target return levels are high, the diversification ratio is not very sensitive to variations of $\Delta L$. Conversely, for low-risk strategies the choice of $\Delta L$ seems to play an important role in the diversification process. Furthermore, for large $\Delta L$ the index $H I(x)$ tends to stabilize at a maximum value. This behavior confirms that diversification depends on several factors, as also stated in Fabozzi and Focardi [11]. 

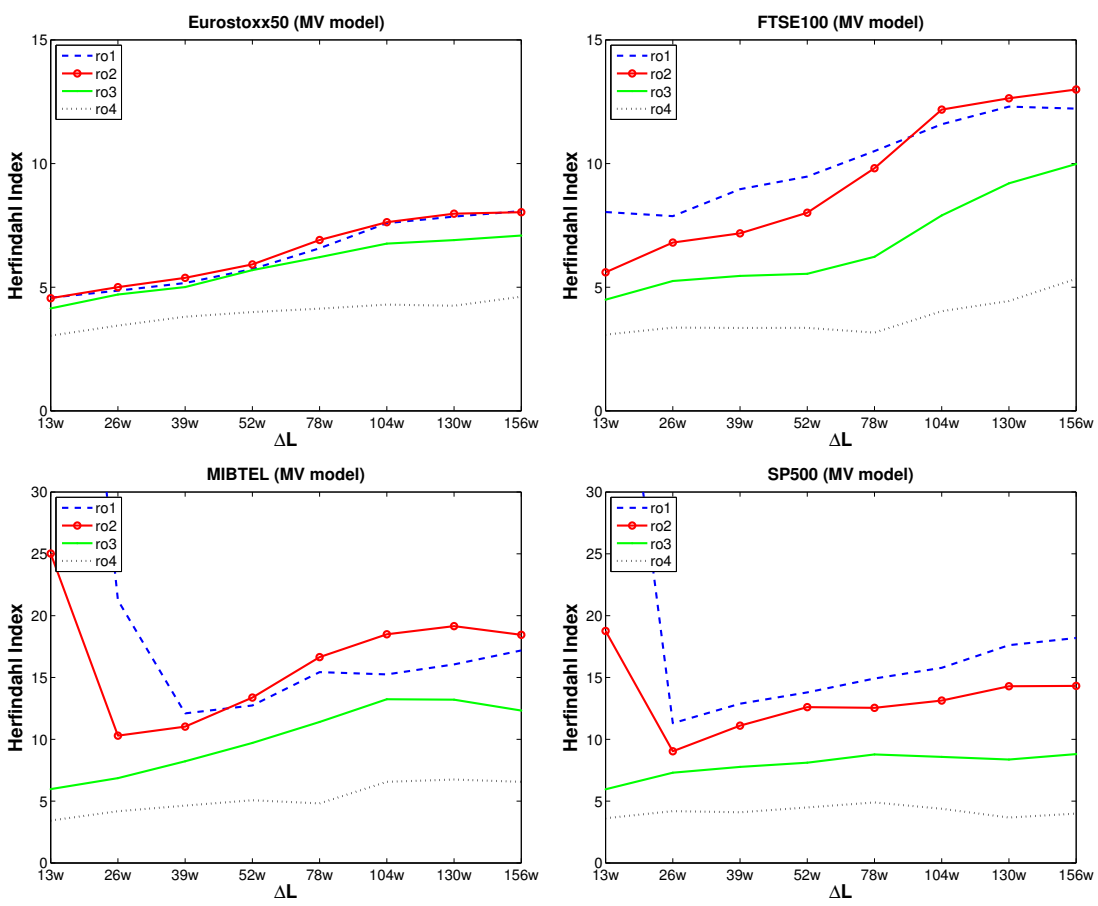

Figure 8: Herfindahl index of the MV efficient portfolios for several target return levels when varying $\Delta L$
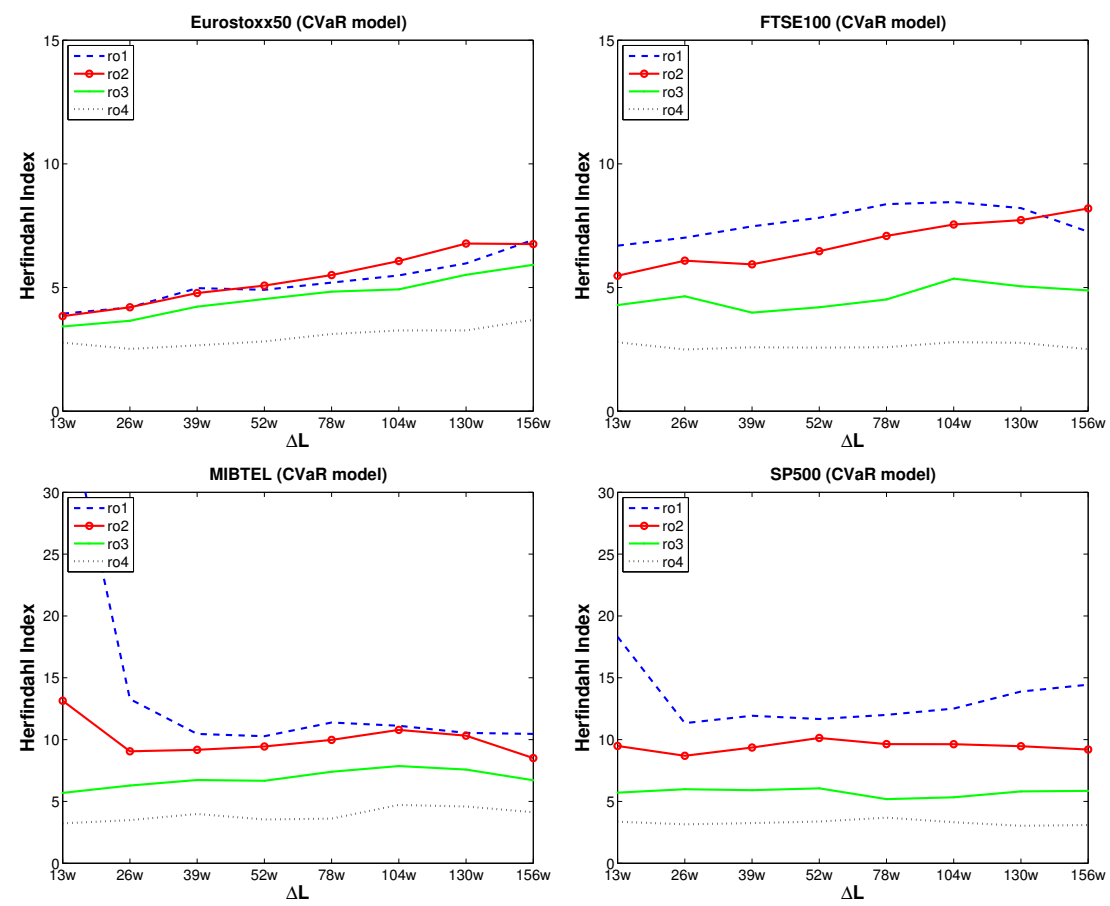

Figure 9: Herfindahl index of the MCVaR efficient portfolios for several target return levels when varying $\Delta L$ 


\section{Concluding remarks}

We have performed an extensive empirical analysis to try to determine the best patterns of in-sample and out-of-sample periods when applying the MeanVariance or the Mean-CVaR models to the portfolio selection problem. In contrast to Ulucan [29] and in agreement with Lee [16], we have observed that for both models the selected portfolios usually have the best performance for holding periods longer than 1 year, while the optimal length of the learning period is between 1 and 2.5 years. However, the benefits of setting a long investment horizon tend to decrease when the learning period increases. We have then compared the performance of the portfolios selected by these models with some benchmarks. For low-risk strategies, the models provide a worse performance than that of the equally-weighted portfolio. For high-risk strategies the MV and the MCVaR models outperform this benchmark, in agreement with DeMiguel et al. [10]. In addition, the performance of the Market Index is typically worse than that of the portfolios constructed by the models. We conclude emphasizing that, as far as we know, this is the first study that analyzes the joint dependence of portfolio performance on the length of both the learning and the holding periods. Of course other factors not considered here may affect the portfolio performance (e.g., the sampling interval of the time series, see Cont [9] and references therein) and they may constitute an interesting subject for further research.

\section{References}

[1] C. Acerbi, Spectral measures of risk: a coherent representation of subjective risk aversion, Journal of Banking \& Finance 26 (2002), 1505-1518.

[2] C. Acerbi and D. Tasche, On the coherence of expected shortfall, Journal of Banking \& Finance 26 (2002), 1487-1503.

[3] A. Adam, M. Houkari, and J.P. Laurent, Spectral risk measures and portfolio selection, Journal of Banking \& Finance 32 (2008), 1870-1882.

[4] P. Artzner, F. Delbaen, J.M. Eber, and D. Heath, Coherent measures of risk, Mathematical finance 9 (1999), 203-228.

[5] C.R. Bacon, Practical portfolio performance measurement and attribution, vol. 546, Wiley, 2008.

[6] V.S. Bawa, Optimal rules for ordering uncertain prospects, Journal of Financial Economics 2 (1975), 95-121. 
[7] M.J. Best and R.R. Grauer, Sensitivity analysis for mean-variance portfolio problems, Management Science 37 (1991), 980-989.

[8] F. Cesarone, A. Scozzari, and F. Tardella, A new method for meanvariance portfolio optimization with cardinality constraints, Annals of Operations Research 205 (2013), no. 1, 213-234.

[9] R. Cont, Empirical properties of asset returns: stylized facts and statistical issues, Quantitative Finance 1 (2001), 223-236.

[10] V. DeMiguel, L. Garlappi, and R. Uppal, Optimal versus naive diversification: How inefficient is the $1 / N$ portfolio strategy?, Review of Financial Studies 22 (2009), 1915-1953.

[11] F. Fabozzi and S. Focardi, Diversification: Should we be Diversifying Trends?, The Journal of Portfolio Management 36 (2010), 1-4.

[12] P.C. Fishburn, Mean-risk analysis with risk associated with below-target returns, The American Economic Review (1977), 116-126.

[13] K. Holmstrom, A.O. Goran, and M.M. Edvall, Users guide for TOMLAB, November 2007.

[14] L.J. Hong and G. Liu, Simulating sensitivities of conditional value at risk, Management Science 55 (2009), 281-293.

[15] H. Konno, Portfolio Optimization using 1 L Risk Function, IHSS Report, Tech. report, Institute of Human and Social Sciences, Tokyo Institute of Technology, 1998.

[16] W.Y. Lee, Diversification and time: Do investment horizons matter?, The Journal of Portfolio Management 16 (1990), no. 3, 21-26.

[17] R. Mansini, W. Ogryczak, and M.G. Speranza, Conditional value at risk and related linear programming models for portfolio optimization, Annals of Operations Research 152 (2007), 227-256.

[18] H. M. Markowitz, Portfolio selection, The Journal of Finance 7 (1952), $77-91$.

[19] _ Portfolio selection: Efficient diversification of investments, Cowles Foundation for Research in Economics at Yale University, Monograph 16, John Wiley \& Sons Inc., New York, 1959.

[20] _ Mean-variance analysis in portfolio choice and capital markets, Basil Blackwell, Oxford, 1987. 
Learning and holding periods for portfolio selection models

[21] A. Meucci, Risk and asset allocation, Springer, 2009.

[22] J. P. Morgan, Riskmetrics-technical document, Tech. report, New York: Morgan Guaranty Trust Company of New York, 1994, 2th ed.

[23] G. C. Pflug, Some remarks on the Value-at-Risk and the Conditional Value-at-Risk, Probabilistic constrained optimization. Methodology and applications (S. P. Uryasev, ed.), Kluwer Academic Publishers, 2000.

[24] R.T. Rockafellar and S. Uryasev, Optimization of Conditional Value-atRisk, Journal of Risk 2 (2000), 21-42.

[25] R.T. Rockafellar, S. Uryasev, and M. Zabarankin, Generalized deviations in risk analysis, Finance and Stochastics 10 (2006), no. 1, 51-74.

[26] A. D. Roy, Safety first and the holding of assets, Econometrica 20 (1952), 431-449.

[27] M.G. Speranza, A heuristic algorithm for a portfolio optimization model applied to the Milan stock market, Computers \& Operations Research 23 (1996), no. 5, 433-441.

[28] D. Tasche, Expected shortfall and beyond, Journal of Banking \& Finance 26 (2002), 1519-1533.

[29] A. Ulucan, An analysis of mean-variance portfolio selection with varying holding periods, Applied Economics 39 (2007), 1399-1407.

\section{Received: July 31, 2013}

